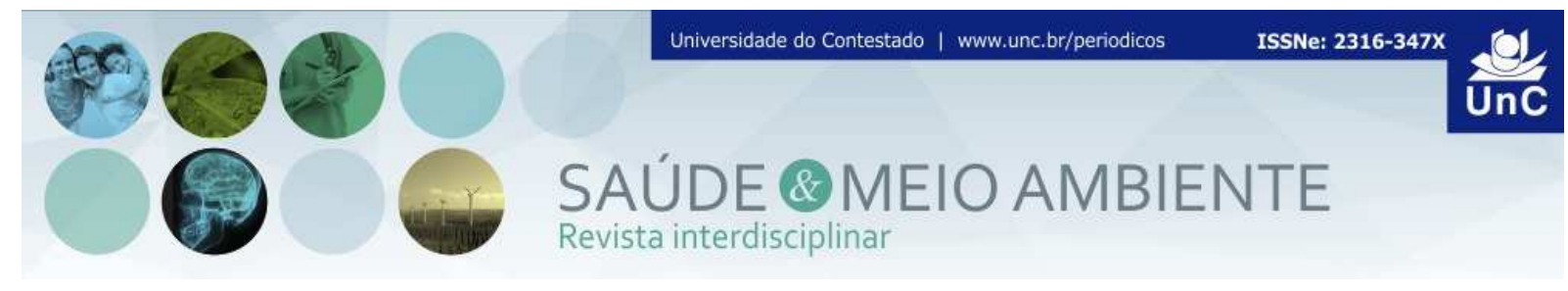

\title{
MUDANÇAS REFRATIVAS EM PÓS-OPERATÓRIO DE PACIENTES SUBMETIDOS À CIRURGIA DE PTERÍGIO PELA TÉCNICA DE TRANSPLANTE CONJUNTIVAL
}

\author{
Bibiana Andrea Pulido Valbuena Pfeiffer ${ }^{1}$ \\ Avelino Nelson Filipe Mazuze ${ }^{2}$ \\ Ronaldo Teixeira Mendes ${ }^{3}$
}

\begin{abstract}
RESUMO
Dentre as alterações patológicas que podem provocar mudanças visuais e refrativas, pode-se citar o pterígio. É uma patologia do segmento anterior do olho, que se caracteriza pelo crescimento do tecido conectivo da conjuntiva bulbar. O objetivo do presente estudo consistiu em verificar se existem mudanças refrativas em pacientes submetidos à cirurgia de pterígio pela técnica de transplante conjuntival. Realizou-se um estudo do tipo exploratório-descritivo e retrospectivo. Para coleta de dados foi feito uma revisão de prontuários de 25 pacientes submetidos à cirurgia de pterígio pela técnica de transplante conjuntival, na Clínica Dr. Canto, na cidade de Curitiba, no estado do Paraná, no período de novembro de 2013 até junho de 2014. As variáveis clínicas analisadas foram ceratometria (meridiano mais plano, meridiano mais curvo e eixo) e refração (esfera, cilindro e eixo). Houve um encurvamento de aproximadamente $0,75 \mathrm{D}$ para meridiano mais plano e de $0,25 \mathrm{D}$ para o meridiano mais curvo e uma redução de aproximadamente 0,61D no astigmatismo corneal. Em relação a refração, para a variável esfera e cilindro houve uma redução de $0,85 \mathrm{D}$. Assim, após análise dos resultados concluiu-se que a cirurgia de pterígio pela técnica de transplante conjuntival produz mudanças refrativas como já era esperado, porém estás mudanças não afetam a visão do paciente.
\end{abstract}

Palavras Chaves: Pterígio. Cirurgia. Mudanças refrativas.

\footnotetext{
1Optómetra, Universidade de La Salle, Bogotá Colombia, Especialista em Gerência em Saúde. Professora na Universidade do Contestado - Campus Canoinhas. Santa catarina. Brasil. E-mail: bibianpulido@hotmail.com

2 Graduado em Optometria pela Universidade do Contestado (UnC Canoinhas - Canoinhas). Também é Licenciado em Ciências Sociais com Ênfase em Desenvolvimento Regional pela Universidade do Contestado (UnC- Campus Canoinhas). Docente do curso de Optometria na Universidade de Lúrio em Moçambique, Campus Marere, Nampula - Moçambique. Membro do Grupo de Pesquisa em Interdisciplinaridade em Ciências Humanas - Cadastrado pelo Cnpq - Pesquisador convidado. Santa Catarina. Brasil. E-mail: mazuzenelson@hotmail.com

${ }^{3}$ Bacharel em Optometria pela Universidade do Contestado - UnC Canoinhas. Santa Catarina. Brasil.. E-mail: rviccenzo@hotmail.com
} 


\title{
POSTOPERATIVE REFRATIVE CHANGES OF PATIENTS SUBMITTED TO PTERIUM SURGERY BY THE CONJUNCTIVE TRANSPLANTATION TECHNIQUE
}

\begin{abstract}
Among the pathological alterations that can cause visual and refractive changes, one can cite the pterygium. It is a pathology of the anterior segment of the eye, characterized by the growth of connective tissue of the conjunctiva bulbar. The objective of the present study was to verify if there are refractive changes in patients undergoing pterygium surgery using the conjunctival transplant technique. An exploratory-descriptive and retrospective study was carried out. To collect data, a review of medical records of 25 patients submitted to pterygium surgery by the conjunctival transplant technique was performed at the Dr. Canto Clinic in the city of Curitiba, in the state of Paraná, from November 2013 to June 2014. The clinical variables analyzed were keratometry (flatter meridian, more curved meridian and axis) and refraction (sphere, cylinder and axis). There was a curvature of approximately $0.75 \mathrm{D}$ for the flatter meridian and $0.25 \mathrm{D}$ for the more curved meridian and a reduction of approximately $0.61 \mathrm{D}$ in corneal astigmatism. In relation to the refraction, for the variable sphere and cylinder there was a reduction of $0.85 \mathrm{D}$. Thus, after analysis of the results it was concluded that pterygium surgery by the conjunctival transplant technique produces refractive changes as expected, but these changes do not affect the patient's vision.
\end{abstract}

Key Words: Pterygium. Surgery. Refractive changes.

\section{INTRODUÇÃO}

Dentre as alterações patológicas que podem provocar mudanças visuais e refrativas, pode-se citar o pterígio. Um crescimento excessivo do tecido fibrovascular em forma de asa, que progressivamente cresce desde o canto interno até a área pupilar, provocando, desta forma, trações que podem levar a mudanças refrativas. Frequentemente, observa-se mais na região nasal que temporal (concretamente na área interpalpebral, entre as $3 \mathrm{~h}$ e $9 \mathrm{~h}$ ). O pterígio pode ser unipolar (afeta apenas uma parte) ou bipolar, quando afeta tanto região temporal assim como a nasal, embora seja geralmente nasal. Eles também podem ser uni ou bilateral (HERNANDEZ, 2008).

A etiologia do pterígio ainda não está completamente explicada. Alguns estudos associam o seu surgimento a diversos fatores como hereditariedade, exposição prolongada aos raios ultravioletas, irritação crônica resultante de condições climáticas adversas, vento e poeira, entre outros (DEVIA; LÓPEZ, 2006).

A intervenção cirúrgica constitui até o momento o único procedimento disponível para a completa resolução do pterígio. É indicada nos casos em que os pacientes sentem um desconforto exacerbado ou ainda quando há prejuízo da acuidade visual, restrição da motilidade ocular, inflamações crônicas, sintomas irritativos persistentes, ou alterações cosméticas (SILVA, 2008). 
A técnica de transplante conjuntival tem sido o procedimento mais utilizado para o tratamento de pterígio devido aos menores índices de complicações em pósoperatório e baixo índice de recidiva, sendo, portanto, o procedimento útil na maioria dos casos.

Estudos sobre as variações visuais e refrativas decorrente da cirurgia de pterígio têm apontado para presença significativa de erros refrativos como a complicação mais comum em pós-operatório de cirurgia de pterígio. Neste sentido, segundo afirma Anduze (2009) a presença de astigmatismo em pós-operatório ocorre quando a membrana de Bowman é destruída pela cabeça do pterígio e assim avançando em direção ao estroma que é invadido pelo tecido fibroso, que, consequentemente, resulta na deformação da curvatura da córnea. Ferraz et al. (2002) analisando as alterações corneanas induzidas pela presença do pterígio em pós-operatório (entre 30 a 60 dias após a cirurgia) observaram que existe uma variação significativa de até 0,50 Dioptrias (D) de astigmatismo nos pterígios de grau I e variação de 0,50-1,50 D nos pterígios de grau II, III e IV, o que permitiu concluir que existem variações da curvatura corneana principalmente nos pterígios maiores (GIII e GIV). Segundo estes autores, essas variações observadas estiveram mais associadas ao tamanho do pterígio do que com a idade dos portadores ou com as características da lesão. Neste sentido, quanto maior é a extensão do pterígio, maior será a ametropia produzida, sobretudo, em casos em que a lesão se estende além de $1,0 \mathrm{~mm}$ do limbo. Portanto, pterígios avançados provocam modificações ceratométricas que são apenas parcialmente corrigidas pela sua exérese, mantendo valores de astigmatismo regular e irregular significativos após a cirurgia.

O objetivo deste estudo consistiu em verificar se existem mudanças refrativas em pacientes submetidos à cirurgia de pterígio pela técnica de transplante conjuntival, procurando, desta forma, analisar as variações produzidas na ceratometria (meridiano mais plano, meridiano mais curvo e eixo) e na refração (esfera, cilindro e eixo).

\section{METODOLOGIA}

A presente pesquisa caracteriza-se como um estudo exploratório-descritivo e retrospectivo.

A coleta de dados foi obtida por meio de uma revisão de prontuários de pacientes submetidos à cirurgia de pterígio pela técnica de transplante conjuntival, na Clínica Dr. Canto, na cidade de Curitiba, no estado do Paraná. Foram analisados prontuários referentes aos meses de janeiro de 2010 à dezembro de 2013. As variáveis clínicas analisadas nos prontuários foram ceratometria (meridiano mais plano, meridiano mais curvo e eixo) e refração (esfera, cilindro e eixo).

Os prontuários foram selecionados com base nos seguintes critérios de inclusão: pacientes maiores de 30 anos de idade, sem distinção de gênero ou raça, de diversas profissões, que foram submetidos à cirurgia de pterígio pela técnica de 
transplante conjuntival e com acompanhamento pós cirúrgico no intervalo de 30 até 120 dias.

Foram excluídos desta pesquisa prontuários incompletos, de pacientes que não fizeram o acompanhamento de forma adequada após a cirurgia, de pacientes com antecedentes de pterígio recidivado, antecedentes de cirurgia refrativa e qualquer patologia ocular que modifique o estado refrativo do paciente.

Os dados obtidos foram catalogados numa ficha de cadastro onde constam sexo, idade e profissão, olho afetado, ceratometria pré e pós operatória e auto refração em pré e pós operatório.

Inicialmente, foi feita uma análise descritiva para a caracterização da amostra segundo as frequências do gênero, idade, olho afetado. Posteriormente, fez-se uma análise dos valores clínicos relacionados as mudanças ceratométricas no meridiano mais plano, meridiano mais curvo e o astigmatismo corneal no pré e pós-operatório; com respeito ao eixo da ceratometria avaliou-se as mudanças em graus e segundo sua posição, foram desconsideradas mudanças de até 1 grau no eixo assim como as córneas esféricas.

Para o estudo das mudanças refrativas do valor esférico e cilíndrico fez-se uma análise das frequências entre pré e pós cirúrgico. As mudanças produzidas no eixo da auto refração foram analisadas segundo a posição e grau, foram desconsiderados pacientes que tiveram mudanças de até 1 grau no eixo da autorefração.

Para o tratamento dos dados usou-se Microsoft Excel 2013 e o programa estatístico SPSS (Statistical Package for Social Sciences) versão 22.0 para apresentação dos gráficos e tabelas em percentagens e em valores absolutos.

Para apontar a significância estatística dos dados pós-cirúrgicos dos valores ceratométricos (astigmatismo corneal, meridiano mais plano, meridiano mais curvo e eixo) e dos valores refrativos (esfera e cilindro), foi aplicado o teste de " $t$ " de Student para dados pareados.

Neste trabalho, utilizou-se o nível de significância $\alpha=5 \%$, e como os dados foram baseados num grupo de 27 olhos então tem-se que o valor crítico $\pm t_{n-1}(1-\alpha / 2)$ é dado por:

$$
t_{26}(1-\alpha / 2)=2,056
$$

Para este tratamento estatístico, temos que o teste é bicaudal, bilateral, assim geram-se duas regiões de rejeição (caudas) e apenas uma região de aceitação, central. Neste sentido, tendo em conta o valor crítico acima apresentado, as regiões de rejeição e aceitação para este trabalho podem ser melhor apresentadas na figura a seguir: 


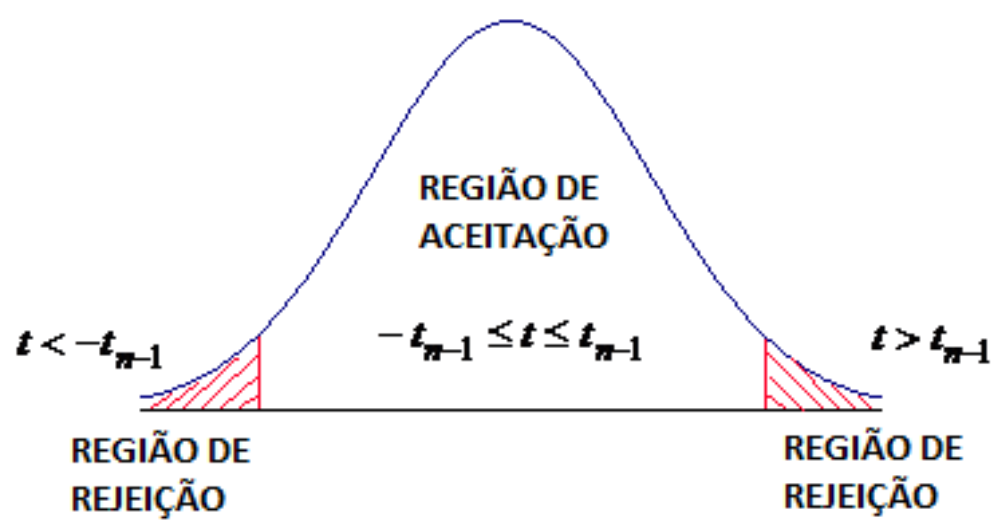

Assim, definimos

Se $t<-t_{n-1}(1-\alpha / 2)$ ou $t>t_{n-1}(1-\alpha / 2)$ rejeita-se a hipótese nula $\left(\mathrm{H}_{0}\right)$, ou seja, rejeita-se a igualdade das médias;

Se $-t_{n-1}(1-\alpha / 2) \leq t \leq t_{n-1}(1-\alpha / 2)$ se aceita a hipótese nula $\left(\mathrm{H}_{0}\right)$, ou seja, se aceita a hipótese de que as médias são iguais.

Com base no valor amostral obtido, a decisão de rejeitar ou aceitar $\mathrm{H}_{0}$ obedece aos seguintes parâmetros: se o valor amostral cair na região de rejeição será recusada à hipótese de que existe uma variação significativa após a cirurgia de pterígio; a decisão de aceitar $\mathrm{H}_{0}$ somente será aceite se o valor amostral cair na região de aceitação (o gráfico acima expressa claramente esta situação).

\section{RESULTADOS E DISCUSSÃO}

Do total da amostra, cerca de $52 \%$ eram mulheres e $48 \%$ homens, sendo o olho esquerdo o mais afetado. A média de idade dos pacientes submetidos a cirurgia de pterígio foi de 50,91 anos. Dos 25 prontuários dos pacientes submetidos a cirurgia cerca de $92 \%$ dos pacientes tiveram pterígio unilateral e $8 \%$ pterígio bilateral. Entretanto, pesquisas brasileiras mostraram acometimento de ambos os sexos, nas mesmas proporções, ou ainda predomínio no sexo feminino.

$\mathrm{Na}$ literatura, pessoas com faixa etária entre 30 a 50 anos de idade são mais suscetíveis em desenvolver a lesão, o que se confirmou na presente pesquisa, sendo que grande parte dos pacientes submetidos a cirurgia de pterígio eram indivíduos acima de 40 anos de idade, sendo que $32 \%$ dos pacientes apresentavam faixa etária no intervalo entre 51 a 60 anos de idade, com uma média de idade de 50,91 anos.

Do total da amostra $92 \%$ dos pacientes tiveram pterígio unilateral e $8 \%$ pterígio bilateral. Estes resultados são compatíveis com os resultados encontrados por Ribeiro 
et al. (2011) num estudo sobre as características e a prevalência de pterígio na comunidade ribeirinha dos Rios Solimões e Jarupá localizados na Amazônia Brasileira. Nesta pesquisa, estes autores verificaram que a faixa etária mais acometida situava-se no intervalo de idade entre 41 à 50 anos, sendo o sexo feminino o grupo mais afetado.

Ainda nesta direção, uma outra pesquisa que evidencia este aspecto foi apresentada por Paes (2010) num estudo sobre o tratamento cirúrgico do pterígio primário: comparação entre as técnicas de rotação de retalho conjuntival e transplante de membrana aminiótica. Segundo este autor, pessoas com faixa etária entre 20 a 50 anos de idade são as mais afetadas, porém sem variabilidade significativa entre os sexos.

Schellini et al (2005) analisando as características de portadores de pterígio na região de Botucatu em São Paulo em aproximadamente 785 pacientes, por meio da relação existente entre exposição a luz solar e a presença da lesão constataram que do total dos pacientes analisados cerca de metade dos portadores de pterígio eram do sexo feminino e com idade superior a 40 anos.

No estudo das variações ceratométricas (gráfico 1) no meridiano mais plano observou-se que no pré-cirúrgico, cerca de $48 \%$ dos olhos da amostra apresentavam o K mais plano nos intervalos entre 40,12 a 43, 00 dioptrias, com uma média de 42,55 D, e no pós-cirúrgico houve um ligeiro encurvamento, portanto cerca de $63 \%$ dos olhos apresentaram o $\mathrm{K}$ mais plano nos intervalos entre 43,12 a 46, 00 dioptrias com a média de 43,32 D. Para o meridiano mais curvo o(gráfico 2) bservou-se que houve uma ligeira variação nas médias pré $(44,37 \mathrm{D})$ e pós-cirúrgica $(44,53 \mathrm{D})$ sendo que nos dois momentos cerca de $81,48 \%$ dos olhos apresentavam o meridiano curvo nos intervalos entre 43,12 a 46, 00 dioptrias. Para variável astigmatismo corneano (gráfico 3) observou-se que a maior parte dos olhos (66,66\%) apresentava astigmatismo corneano no intervalo de 0,25 a 2,00Dpts, após a cirurgia esta porcentagem aumento para $96,29 \%$ neste mesmo intervalo, portanto, houve uma diminuição do astigmatismo corneano pós cirúrgico.

Gráfico 1 - Variações ceratométricas no meridiano mais plano

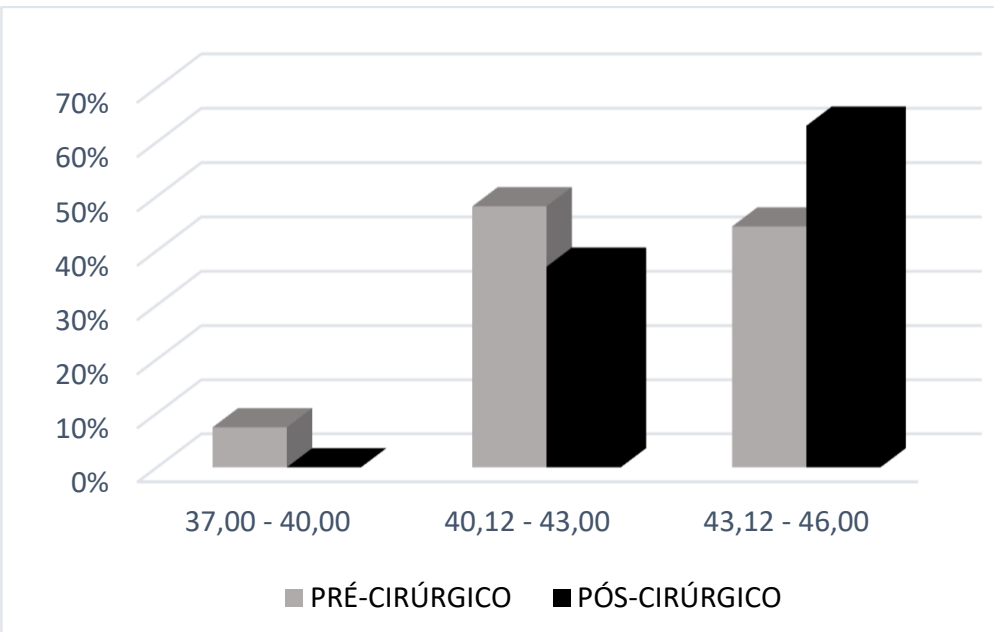

Saúde Meio Ambient. v. 6, n. 2, p. 94-107, jul./dez. 2017 
Gráfico 2 - Variações ceratométricas no meridiano mais curvo

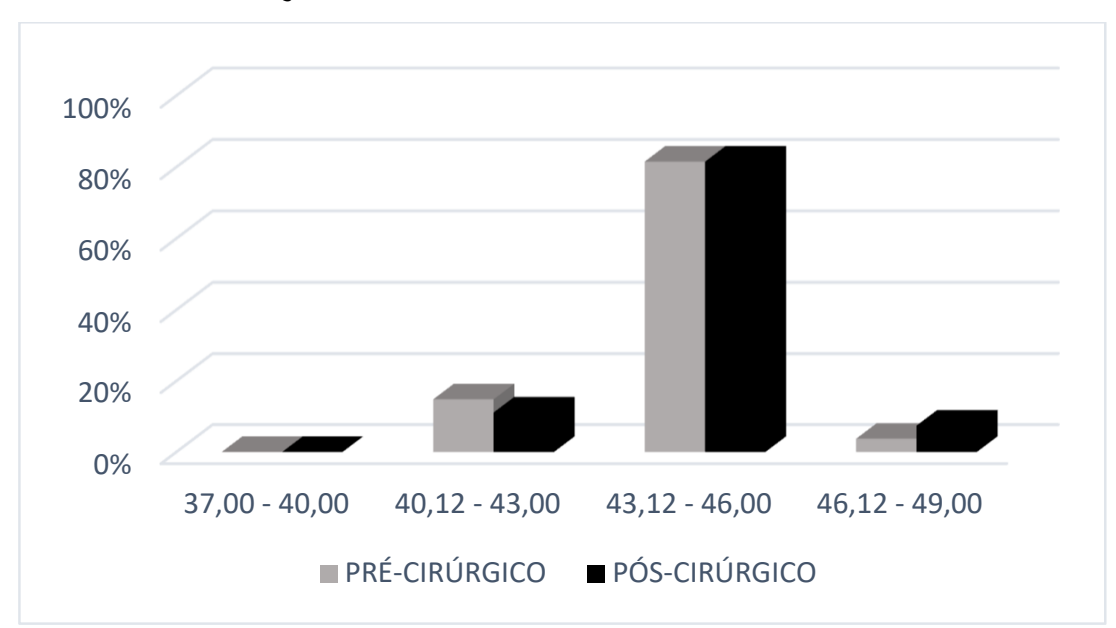

Gráfico 3 - Variações do astigmatismo corneano

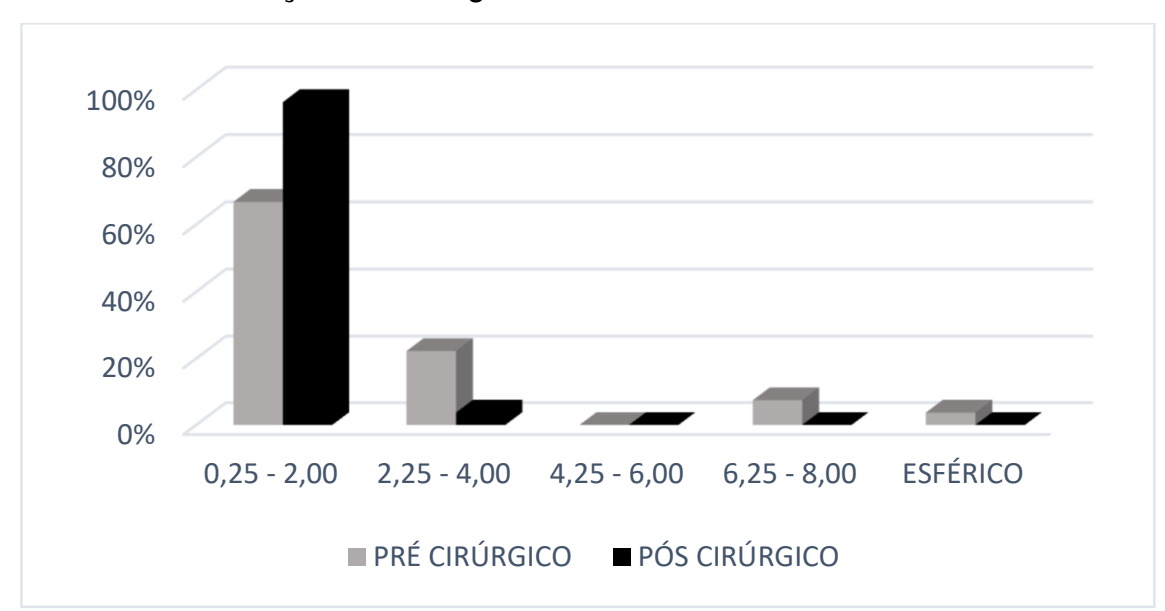

Em relação as variações refrativas produzidas no valor esférico (gráfico 4) observaram-se que cerca de $70 \%$ do total da amostra apresentavam antes da cirurgia de pterígio o valor da esfera no intervalo entre $-0,75$ a 1,25Dpts, após houve um aumento neste intervalo, passando para $81 \%$, o que implica dizer que houve uma redução no valor da esfera. Para o valor do cilindro refrativo (gráfico 5) observou-se que antes da cirurgia $55 \%$ dos olhos apresentavam o valor do cilindro nos intervalos entre PLANO a $-0,75 \mathrm{D}$, tendo após a cirurgia aumentando para $74 \%$, portanto houve uma redução do valor do cilindro após a cirurgia de pterígio. Também se observou que antes da cirurgia de pterígio a maioria dos olhos apresentavam astigmatismo a favor da regra (WR) (44\%), após a cirurgia de pterígio houve uma ligeira diminuição do WR e um ligeiro aumento de astigmatismo obliquo que era $11 \%$ e passou para $25 \%$ no pós cirúrgico. 
Mudanças refrativas em pós-operatório de pacientes submetidos à cirurgia de pterígio pela técnica de transplante conjuntival

Gráfico 4 - Variações no valor refrativo esférico

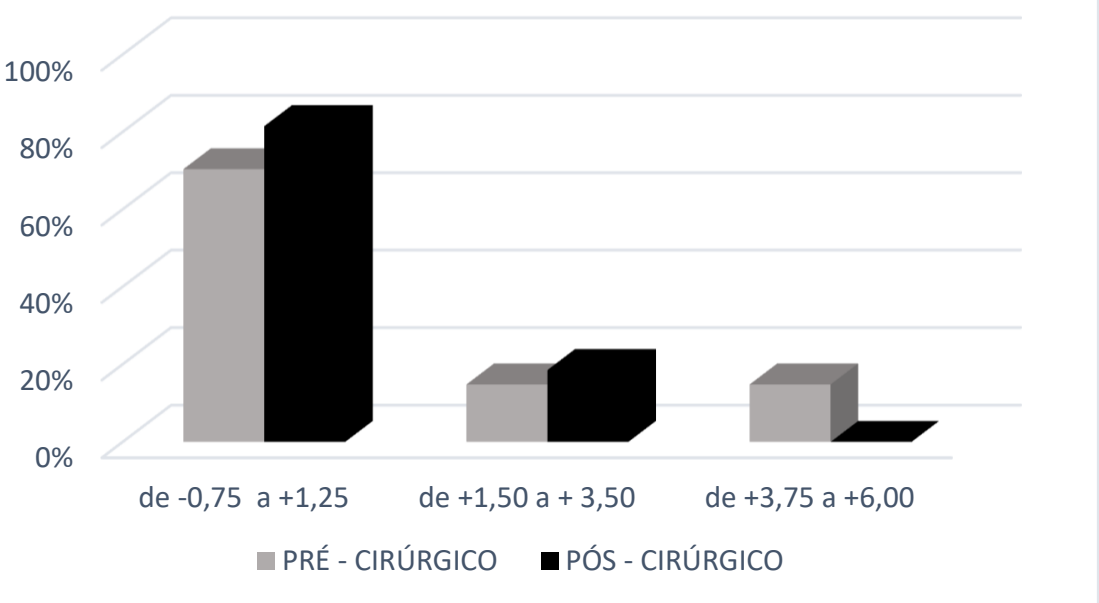

Gráfico 5 - Variações refrativas no valor do cilindro

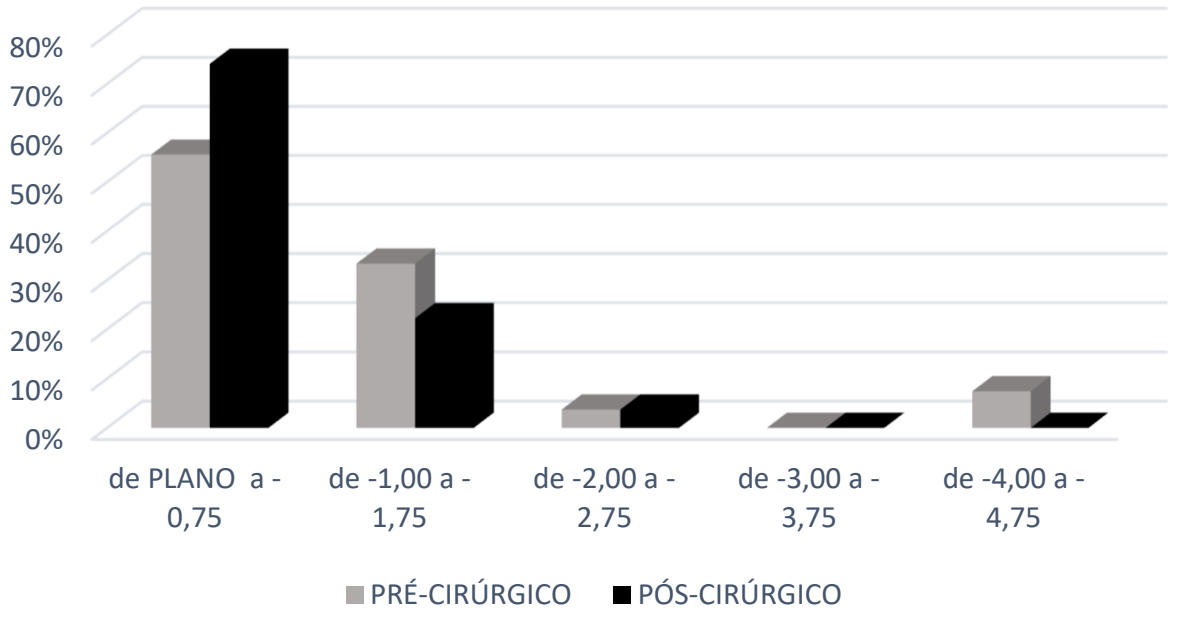


Gráfico 6 - Variações do segundo a posição do eixo

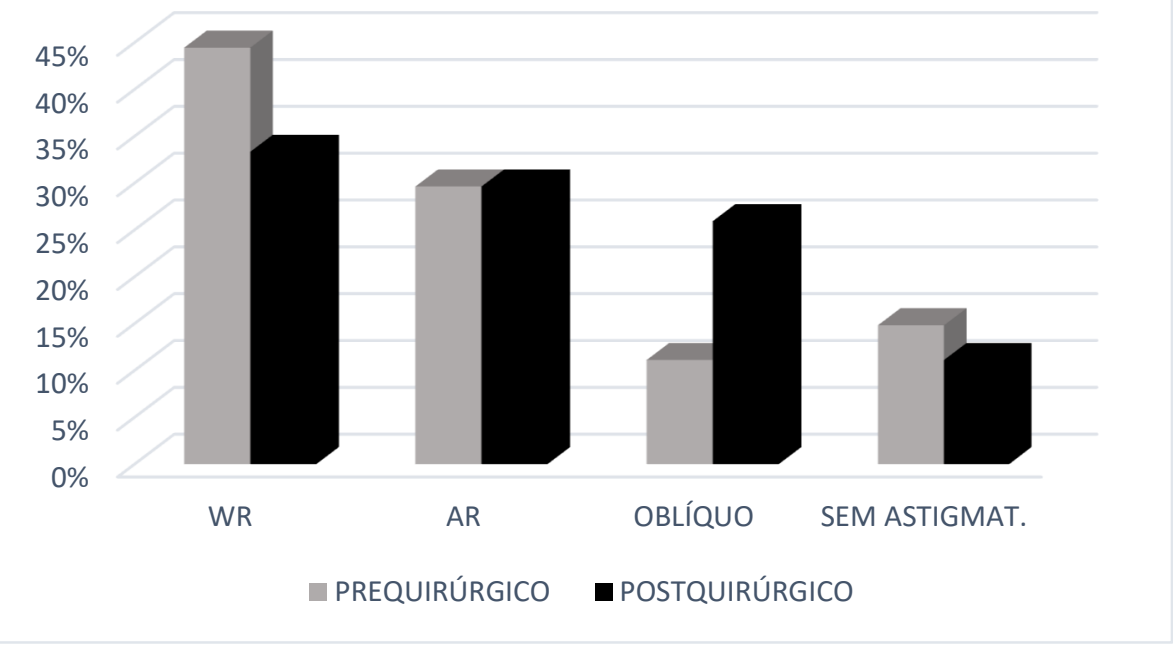

Para a análise comparativa foi utilizada o teste de $t$ Student entre os dados antes e após a cirurgia, para as cinco características analisadas, descritas nas tabelas a seguir

Tabela 1 - Análise estatística das médias pré e pós-operatório dos valores da ceratometria para o meridiano mais plano, meridiano mais curvo e astigmatismo corneano.

\begin{tabular}{l|l|l|l|l|l}
\hline & \multicolumn{5}{|l|}{ CERATOMETRIA } \\
\cline { 2 - 6 } & Média Pré & Média Pós & $\begin{array}{l}\text { Média } \\
\text { diferença }\end{array}$ & $\begin{array}{l}\text { Desvio padrão da } \\
\text { diferença }\end{array}$ & $\begin{array}{l}\text { Estatística } \\
t\end{array}$ \\
\hline Mais Plano & 42,556 & 43,324 & $-0,769$ & 1,189 & $-3,359$ \\
\hline Mais Curvo & 44,380 & 44,537 & $-0,157$ & 0,368 & $-2,223$ \\
\hline Ast. corne. & 1,824 & 1,213 & 0,611 & 1,303 & 2,436 \\
\hline
\end{tabular}

Analisando o valor da estatística $t$ para cada uma das variáveis ceratometricas temos: para o meridiano mais plano $(\mathrm{K})$, meridiano mais curvo, eixo e astigmatismo corneano, concluiu-se que para $\mathrm{K}$ mais plano houve um encurvamento de aproximadamente $0,75 \mathrm{D}$, enquanto que para $\mathrm{K}$ mais curvo observou-se um ligeiro encurvamento de $0,25 \mathrm{D}$. Embora tenham sido visíveis as diferenças entre o préoperatório e o pós-operatório nas variáveis (meridiano mais plano e meridiano mais curvo), foi necessária a análise estatística, através do teste $t \mathrm{e}$, segundo os resultados concluiu-se que: no meridiano mais plano, a estatística resultou em $t=-3,359<-2,056$ e, portanto, para o nível de $\alpha=5 \%$, existiram diferenças estatisticamente significativas; o mesmo comportamento observou-se para o meridiano mais curvo, onde a estatística resultou em $t=-2,223<-2,056$, e assim foi comprovada para o nível de $\alpha=5 \%$, que existiram diferenças significativas nas médias entre o pré e pósoperatório. 
Mudanças refrativas em pós-operatório de pacientes submetidos à cirurgia de pterígio pela técnica de transplante conjuntival

Estes resultados são compatíveis com os resultados apresentados por Maheshwaris (2007) apud Figueroa (2012) num estudo sobre as mudanças refrativas produzidas pela cirurgia de pterígio e, segundo este autor, pacientes que apresentam um encurvamento acentuado no meridiano mais plano e um ligeiro encurvamento no meridiano mais curvo provavelmente são portadores de pterígios avançados.

No astigmatismo corneano observou-se que $66,66 \%$ do total dos pacientes da amostra para o pré-cirúrgico, apresentaram astigmatismos corneanos de até 2.00D e após a cirurgia esta porcentagem aumentou para 96,29\%, revelando uma diminuição do astigmatismo corneano após a cirurgia de pterígio, tendo a média antes da cirurgia de 1,82D e a após a cirurgia de 0,61D. O Teste estatístico apresentou-se em $t=2,436>2,056$ logo houve diferenças significativamente estatísticas nas médias.

A alteração topográfica mais encontrada, associada à presença do pterigio é o astigmatismo assimétrico a favor da regra, causado pelo aplanamento da córnea na direção da lesão. Entre os mecanismos propostos para explicar a indução deste, estão a teoria do acúmulo de filme lacrimal no seu ápice devido à irregularidade corneana por ele induzida e a teoria da tração mecânica exercida pela lesão na córnea.

Vives et al. (2013) analisando as mudanças topográficas em pacientes submetidos a cirurgia de pterígio pela técnica de transplante autólogo da conjuntiva concluíram que a cirurgia de pterígio pela técnica de transplante autólogo da conjuntiva reduz o valor do astigmatismo corneano ( uma redução de 1,75-1,25 D no primeiro mês e 1,00 D nos 3 meses posteriores). Ainda, que o astigmatismo encontrado no pré-operatório está associado ao tamanho do pterígio. Também concluíram que existe uma diferença estatisticamente significativa entre o eixo médio do astigmatismo do pré, um mês pós-cirurgia e 3 meses após a cirurgia. Para estes autores, todas essas mudanças encontradas ocorreram logo após a cirurgia (1 mês depois).

Tabela 2 - Análise estatística das médias pré e pós-operatório dos valores da refração para a esfera e cilindro.

\begin{tabular}{l|l|l|l|l|l}
\hline & \multicolumn{4}{|l}{ REFRAÇÃO } & \multicolumn{4}{l}{} \\
& Média Pré & Média Pós & $\begin{array}{l}\text { Média } \\
\text { diferença }\end{array}$ & $\begin{array}{l}\text { Desvio padrão da } \\
\text { diferença }\end{array}$ & $\begin{array}{l}\text { Estatística } \\
t\end{array}$ \\
\hline Esfera & 1,361 & 0,509 & 0,852 & 1,219 & 3,630 \\
\hline Cilíndrico & $-1,009$ & $-0,676$ & $-0,333$ & 0,838 & $-2,067$ \\
\hline
\end{tabular}

Analisando $o$ valor da estatística $t$ para cada uma das variáveis refrativas temos: para a variável esfera, a estatística resultou em $t=3,630>2,056$ logo confirmou-se a existência de alteração significativa estatísticamente na esfera entre as médias para o nível de $\alpha=5 \%$, enquanto que para a variável cilíndro, a estatística resultou em $t=-2,069<-2,056$ logo, também confirmou-se a hipótese da existência de alteração estatisticamente significativa entre as médias para o nível de $\alpha=5 \%$. 
Os resultados desta pesquisa confirmaram com as observações feitas por Errais et al (2013) em um estudo sobre o efeito da cirurgia de pterígio na topográfica de córnea, desenvolvido em 20 olhos. Segundos estes autores, as alterações topográficas produzidas na curvatura cornena e na refração pela cirurgia de pterígio são "quase" reversíveis após tratamento cirúrgico, sobretudo em pacientes submetidos a cirurgia de pterígio pela técnica de transplante conjuntival, uma vez que redução do astigmatismo topográfico e o achatamento da córnea é significativa. No entanto, a previsão destas alterações na refração nem sempre é precisa e, portanto, é possível que haja tanto um aplanamento assim como um encurvamento da córnea, porém com redução de astigmatismo refrativo e a favor da regra.

Outra explicação para estas variações refrativas foi apresentada por Anduze (2009) e segundo este autor, as modificações produzidas na curvatura corneana e, consequentemente na refração são devido a: teoria da elasticidade da córnea; a resistência inerente às forças mecânicas e a duração do esforço dessas forças, que normalmente achata a córnea no meridiano horizontal enquanto que a força da contratura achata a córnea verticalmente no meridiano de $90^{\circ}$.

Ferraz et al. (2002) analisando as alterações corneanas induzidas pela presença do pterígio em pós-operatório (entre 30 a 60 dias após a cirurgia) observaram que existe uma variação significativa de até $0,50 \mathrm{D}$ de astigmatismo nos pterígios de grau I e variação de 0,50-1,50 D nos pterígios de grau II, III e IV, o que permitiu concluir que existem variações da curvatura corneana principalmente nos pterígios maiores (GIII e GIV). Segundo estes autores, essas variações observadas estiveram mais associadas ao tamanho do pterígio do que com a idade dos portadores ou com as características da lesão

Da mesma forma no estudo de Tomikodoro et al (2000) apud Figueroa (2012) demonstrou em 136 pacientes que o tamanho do pterígio se correlaciona significativamente com o poder esférico e o astigmatismo; encontraram também que a cirurgia aumentou em forma apreciável o poder esférico da córnea enquanto que o astigmatismo diminuiu notoriamente.

$\mathrm{Na}$ análise do eixo refrativo, concluiu-se que após a cirurgia de pterígio pela técnica de transplante houve um predomínio de astigmatismo a favor da regra, porém um ligeiro aumento de astigmatismo oblíquo foi constatado. Uma explicação para esta observação foi apresentada por Avisar et ál (2000) apud Figueroa (2012), em um estudo desenvolvido em 94 pacientes, onde se refere que o pterígio primário induze astigmatismos a favor da regra (WR) já pterígios severos induzem mudanças significativas no astigmatismo e eixo, porém observando maiores mudanças no eixo.

Estes resultados são compatíveis com os apresentado por Taylor (2000) num estudo sobre as variações refrativas em pós operatório de pacientes submetidos a cirurgia de pterígio e, segundo este autor, o astigmatismo induzido pela cirurgia de pterígio é na maioria dos casos assimétricos e a favor da regra.

Assim, verifica-se novamente para as duas características que a hipótese de que não houve variação na média das observações deve ser descartada. Porém, para a variável cilindro, a estatística amostral obteve um valor muito próximo ao $t$ crítico, 
assim, caso um novo teste seja feito, com um nível de significância $\alpha<5 \%$, existe uma probabilidade muito grande de que a estatística caia na região de aceitação, e assim assume-se como verdadeira a hipótese nula, de que não existe diferença significativa nas médias, pré o pós operatória.

\section{CONSIDERAÇÕES FINAIS}

Assim concluiu-se que após a cirurgia de pterígio pela técnica de transplante conjuntival houve um encurvamento da córnea, predominância de astigmatismo a favor da regra e tendência a miopização. Portanto, para a variável ceratometria houve um encurvamento de aproximadamente $0,75 \mathrm{D}$ para meridiano mais plano e de $0,25 \mathrm{D}$ para o meridiano mais curvo e uma redução de aproximadamente 0,61D no astigmatismo corneal. Sendo que nas três variáveis existiram diferencias estatisticamente significativas.

Em relação a refração, para a variável esfera e cilindro houve uma redução de 0,85D, tendo antes da cirurgia a média sido de 1,36D e após a cirurgia ficou com 0,50D; o mesmo observou-se para o valor do cilindro, onde houve uma redução de $0,33 \mathrm{D}$, tendo antes da cirurgia a média sido de 1,01D e após a cirurgia passou para 0,67D. Sendo que para as duas variáveis existiram diferenças estatisticamente significativas.

\section{REFERÊNCIAS}

AMARAL, Luiz Antonio Carvalho; VIEIRA, CLÁUDIO Amorim; HADDAD, Luciano H.; MEYER, Rosana D.; HISSA; Lígia. Betaterapia, em doses fracionadas, na prevenção da recidiva do pterígio. Acta Cirúrgica Brasileira, v. 11, n. 4, 1996.

ANDUZE, Alfred L. Pterygium: a pratical guide to management. 1 ed. India: Jaypee Brothers Medical Publishers, 2009.

COUTTS, Sophie Joanna; COOMBES, Andrew. Pterígio: prevalência e gravidade em um cenário oftálmico na Amazônia, Brasil. Revista Brasileira de Oftalmologia. v. 6, p. 372-6, 2012.

COPELAND Jr. Robert ; AFSHARI, Natalie A. Principles and Pratice of Cornea. 1 ed. India: Jaypee Brothers Medical Publishers, 2013.

DíAZ, Alejandro Arias et al. Comportamiento del pterigión según la exposición a radiaciones ultravioletas y sus cambios histológicos de acuerdo con su severidad.

Revista Cubana Oftalmologia v. 22. n. 2. 2009. 
DEVIA, Giovanna; LÓPEZ, Isabel López. Determinación retrospectiva de la prevalencia de pterigio en la población que consultó en el servicio de optometría e oftalmología de el hospital El 'Salvador' Ubaté, durante el 23 Julio 2005 y el 25 de Enejo de 2006. Monografia. Universidade de la Salle Faculdade de Optometria. Bogota. Colombia, 2006.

ERRAIS, K.; BOUDEN, J.; MILI-BOUSSEN, I.; ANANE, R.; BELTAIF, O.; OUERTANI, A. Medded. Effect of pterygium surgery on corneal topography. European Journal of Ophthalmology, v. 18, n. 2, p. 177-181, 2008.

FERRAZ, Fábio Henrique Silva; SCHELLINI, Silva Artioli; HOYANA, Erika; BERNANDES, Suely Romano; PADOVANI; Carlos Roberto. Pterigio e alterações da curvatura corneana. Arquivos Brasileiros de Oftalmologia. São Paulo, v. 65, n. 5, Sept./Oct. 2002.

FIGUEROA; Maria Fernanda Rios. Cambios en la agudeza visual, la queratometría y refracción en pacientes después de cirugía de pterigion grado i y grado ii. Ciencia y tecnologia en salud Visual y ocular, v. 10, n. 1, enero/jun. 2012.

HERNANDEZ, Ivan Dario Castillo. Prevalencia de pterigio en pacientes que asisten al Hospital Rafael Uribe Uribe entre Julio Diciembre del 2007. Monografia. Universidade de la Salle -Faculdade de Optometria. Bogota. Colômbia, 2008.

KWITKO, Sérgio. Transplante da conjuntiva. Arquivos Brasileiros de Oftalmologia. v. 63, n.4, ago. 2000

MAHESHWARI, Pterygium-Induced Corneal Refractive Changes. Indian Journal of Ophthalmology. v. 55, n.5, p. 383-386.

PAES, Joaquim Pereira. Tratamento cirúrgico do pterígio primário: comparação entre as técnicas de rotação de retalho conjuntival e transplante de membrana aminiótica. Tese de Doutorado. Universidade Estadual de Campinas - Faculdade de Ciências Médicas. Campinas, 2010.

PAGNIN, André Franco.Identificação e Caracterização de Pterígio Utilizando Floresta de Caminhos Ótimos e Técnicas de Otimização. Dissertação de Mestrado. Instituto de Biociências, Letras e Ciências Exatas da UNESP de São José do Rio Preto. São Paulo, 2011.

PÉREZ, Joaquim Fernández ; FERNÁNDEZ, Félix Jesús Alañón ; LÓPEZ, Sebastián Ferreiro. Oftalmología en atención primaria.FORMACIÓN ALCALÁ. 2001.

RIBEIRO, Lívia Adnet et al. Características e prevalência do pterígio em comunidades ribeirinhas dos Rios Solimões e Japurá localizados na Amazônia Brasileira. Rev Bras Oftalmologia, v. 70, n. 6, p. 358-62, 2011.

REIS, Ana do Carmo Paula Pessoa dos et al. Detecção de tracoma e doenças corneanas em índios da região do Alto Rio Negro. Arquivos Brasileiros de Oftalmologia. São Paulo, v.65 n.1 jan./fev. 2002. 
SCHELLINI, Silvana Artioli; VELOSO, Carlos Eduardo dos Reis ; LOPES, Wannessa ; PADOVANI, Carlos Roberto Pereira. Características de portadores de pterígio na região de Botucatu. Arquivos Brasileiros de Oftalmologia, v. 68, n. 3, p. 291-4, 2005.

SANDRES, Admsterdan; NETO-TAVARES, José. Epidemiologia do Pterígio em uma Localidade da Amazônia Ocidental do Brasil.Gazeta Médica. Bahia, v. 76, n. 1 p. 2-13, jan./jun. 2006.

SILVA, Ana Paula Martins. Pterígio: uma abordagem atualizada dos métodos de tratamento e da importância da fotoproteção ocular. Monografia. Escola de Saúde do Exército, Programa de Pós-Graduação em Aplicações Complementares às Ciências Militares. Rio de Janeiro, 2008.

TAYLOR, Hugh R. Pterygium. Canada: Kugler Publications, 2000.

VAUGHAN, Daniel G; ASBURY, Taylor; RIORDAN-EVA, Paul. Oftalmologia geral. 4.ed. São Paulo: Atheneu, 2003.

VIVES, Pere Pujol et al. Topographic corneal changes in astigmatism due to pterygium's limbal-conjunctival autograft surgery. Journal of emmetropia, v. 4, jan./mar., 2013.

YAYCIOGLU-ATLAN, Rana et al. Astigmatic changes following pterygium removal: Comparison of 5 different methods. Indian Journal of Ophthalmology, v. 61, n. 3 , mar. 2012.

Artigo recebido em: 22/05/2017

Artigo aprovado em: 15/11/2017

Artigo publicado em: 20/12/2017 